\title{
Reviewer acknowledgement 2016
}

Thomas Liehr ${ }^{1 *}$, Henry Heng ${ }^{2^{*}}$, Yuri Yurov ${ }^{3^{*}}$, Aurelia Meloni-Ehrig ${ }^{4}$ and Ivan lourov ${ }^{3^{*}}$

\section{Contributing reviewers}

The Editors of Molecular Cytogenetics would like to thank all our reviewers who have contributed to the journal in volume 8 (2015).

Dilek Aktas

Turkey

Mara Almeida

Brazil

Daniela Alosi

Denmark

Gilda Alves

Brazil

Lucilene Arilho Ribeiro-Bicudo

Brazil

Rouben Aroutiounian

Armenia

István Balogh

Hungary

Liming Bao

USA

John Barber

UK

Iris Bartels

Germany

Samarth Bhatt

USA

Douglas Bittel

USA

Maria Bonaglia

Italy
Francesco Brancati

Italy

Lukrecija Brecevic

Croatia

Helene Bruyere

Canada

Joern Bullerdiek

Germany

Vincent Cantagrel

France

Isabel Maria Marques Carreira

Portugal

Chiara Castronovo

Italy

Arunrat Chaveerach

Thailand

Sau Wai Cheung

USA

Richard Choy

Hong Kong

Marcelo Cioffi

Brazil

Carlos Cordova-Fletes

Mexico

Luciana da Silva

Spain

\author{
Xiangpeng Dai \\ USA \\ Zunyan Dai \\ USA \\ Josef Davidsson \\ Sweden \\ Angelika Dawson \\ Canada
}

Marc De Braekeleer

France

Edivaldo De Oliveira

Brazil

Marie Dell' Aquila

USA

Jan Diblik

Czech Republic

Regen Drouin

Canada

Peter Duesberg

USA

Andreas Dufke

Germany

Emel Ergul

Turkey

Carmen Esmer

Mexico

*Correspondence: i8lith@mti.uni-jena.de; hheng@med.wayne.edu; y_yurov@

yahoo.com; ivan.iourov@gmail.com

${ }^{1}$ Jena University Hospital, Institute of Human Genetics, Jena, Germany

${ }^{2}$ Center for Molecular Medicine and Genetics, Wayne State University School

of Medicine, Detroit, MI, USA

${ }^{3}$ National Research Center of Mental Health, Moscow, Russia

${ }^{4}$ CSI Laboratories, Alpharetta, USA

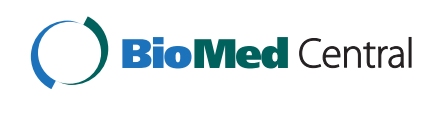


Raffaele Falsaperla

Italy

Fangpu Fan

China

Amanda Figueiredo

Brazil

Christopher Franklin

UK

Catherine French

United States

Radmila Frydrychova

Czech Republic

Helen Fryssira

Greece

M Fukami

Japan

Ioannis Georgiou

Greece

Ad Geurts Van Kessel

Netherlands

Alexander Graphodatsky

Russia

Francesca Grati

Italy

Renzo Guerrini

Italy

Roberta Guilherme

Brazil

Oskar Haas

Austria

Claudia Haferlach

Germany

Ahmed Hamid

Denmark

Karen Harrison

Canada

Nyla Heerema

USA

Walter Hittelman

USA

Ron Hochstenbach

Netherlands

Jennelle Hodge

USA
Steven Horne

USA

Andreas Houben

Germany

Galina Hovhannisyan

Armenia

Maj Hulten

UK

Colleen Jackson-Cook

USA

X Ji

China

John Kamholz

USA

Benjamin Kamien

Australia

Elena Kirillova

Russia

Genevieve Konopka

United States

Nadezda Kosyakova

Germany

Dieter Kotzot

Austria

Victor Kravetz

Russia

Michał Książkiewicz

Poland

Anver Kuliev

USA

Ingo Kurth

Germany

Kwok-Yin Leung

Hong Kong

Peining Li

USA

Shibo Li

USA

Thomas Liehr

Germany

Nestor L Lopez Corrales

Germany

Isabel López-Expósito

Spain
Caroline Mackie Ogilvie

UK

Emmanouil Manolakos

Greece

Marina Manvelyan

Armenia

Ludmila Matyakhina

USA

Cristina Mecucci

Italy

Aurelia Meloni-Ehrig

USA

Martina Merkas

Croatia

Patricia Miron

USA

Hasmik Mkrtchyan

Armenia

Amal Mahmoud Mohamed

Egypt

Wagner Molina

Brazil

Elisabeth Nacheva

UK

Rizwan Naeem

USA

Indrajit Nanda

Germany

Hiroyuki Nawa

Japan

Juergen Neesen

Austria

Heike Nelle

Germany

Beata Nowakowska

Belgium

Moneeb Othman

Germany

Hong Pan

China

Davide Pareyson

Italy

Francesco Pasquali

Italy 
Lorenza Pecciarini

Italy

Michael B. Petersen

Greece

Petr Ráb

Czech Republic

Maryam Rafati

Iran

Martina Rincic

Croatia

Anna Rita Rossi

Italy

Elena Rossi

Italy

Feride Sahin

Turkey

Heather Sanders

USA

Damien Sanlaville

France

Heide Schatten

USA

Albert Schinzel

Switzerland

Brigitte Schlegelberger

Germany

Samantha Schrier Vergano USA
Marco Seri

Italy

Harsh Sheth

UK

Frenny Sheth

India

Malte Spielmann

Germany

Jeremy Squire

Brazil

Pawel Stankiewicz

USA

Zornitza Stark

Australia

Maria Syrrou

Greece

Minghan Tong

China

Vladimir Trifonov

Russia

Angelo Valetto

Italy

Joris Vermeesch

Belgium

Sonja Vernes

Netherlands
E. M. Vestergaard

Denmark

Victoria Voinova

Russia

Emanuela Volpi

UK

Svetlana Vorsanova

Russia

Xinjing Wang

USA

Dorothy Warburton

USA

Jingly Weier

USA

Anja Weise

Germany

Kathleen Wilhelm

Germany

Dan Xu

USA

Zhihong Yang

USA

Cassia Yano

Brazil

Adnan Younis

South Korea 